\title{
Torsade de pointes during low-dosage sotalol therapy in haemodialysis patients
}

\author{
U. Huynh-Do ${ }^{1,2}$, C. Wahl ${ }^{1,2}$, M. Sulzer ${ }^{2}$, H. Bühler ${ }^{2}$ and G. Keusch ${ }^{1}$ \\ ${ }^{1}$ Division of Nephrology and ${ }^{2}$ Medical Clinic, City Hospital Waid, Zürich, Switzerland
}

Key words: haemodialysis; sotalol; ventricular tachycardia; torsade de pointes

\section{Introduction}

Sotalol is the prototype class III agent that combines $\beta$-blocking properties with the propensity to prolong the effective refractory period by lengthening the action potential duration. A review of clinical trials demonstrates that sotalol is an effective treatment for unsustained and sustained ventricular tachyarrhythmias. It is also active against several supraventricular arrhythmias. Sotalol is safe and generally well tolerated; most of the adverse effects experienced with its use are related to its $\beta$-blocking activity. However, sotalol has been associated with life-threatening proarrhythmias, including torsade de pointes, although the incidence of this adverse effect is low, varying between 1.8 and $3 \%$ in the clinical trials conducted to date [1-3]. To our knowledge, the occurrence of sotalol-induced torsade de pointes has never been reported before in haemodialysis (HD) patients. We report here three HD patients who underwent cardiopulmonary resuscitation for torsade de pointes and cardiopulmonary arrest under low-dosage sotalol therapy.

\section{Case reports}

Case 1. A 53-year-old female patient had had recurrent pulmonary infections because of bronchiectasis of the lower lobes from the age of 19. HD was started in 1983 because of end-stage renal disease (ESRD) due to secondary renal amyloidosis. In November 1992, a resection of the lingula was performed. Postoperatively sotalol was started in a low dosage of $2 \times 40 \mathrm{mg} /$ day for recurrent supraventricular tachyarrhythmias. On the second day, the patient underwent mechanical cardiopulmonary resuscitation because of torsade de pointes. The ECG showed a prolonged corrected QTc

Correspondence and offprint requests to: $\mathrm{U}$. Huynh-Do, MD, Division of Nephrology and Medical Clinic, City Hospital Waid, Tièchestrasse 99, CH-8037 Zürich, Switzerland. interval of $550 \mathrm{~ms}$. Sotalol was stopped and magnesium sulphate was given; the QTc interval normalized and the patient remained in a stable sinus rhythm of $70 / \mathrm{min}$.

Case 2. A 78-year-old male patient with a known hypertensive and coronary heart disease had been on HD since 1979 because of ischaemic nephropathy. In July 1995 he developed intermittent supraventricular tachyarrhythmias; because of digoxin incompatibility, sotalol was given in a dosage of $2 \times 40 \mathrm{mg} / \mathrm{day}$ without supplemental dosage after HD. One month later he was hospitalized because of acute gastrointestinal bleeding under aspirin and indomethacin. During the first 3 days he needed daily blood transfusions and endoscopic haemostasis because of recurrent bleeding. On the third day, torsade de pointes with haemodynamic compromise occurred twice (Figure 1) but responded promptly to cardioversion and magnesium sulphate infusion. The ECG showed a bradycardia of $50 / \mathrm{min}$ and a slightly prolonged QTc of $480 \mathrm{~ms}$. Sotalol was replaced by atenolol and the patient recovered uneventfully.

Case 3. A 73-year-old female patient with hypertensive, valvular and coronary heart disease had been on CAPD, then HD since 1979 because of analgesicassociated nephropathy, In May 1995 she underwent parathyroidectomy for secondary hyperparathyroidism. Postoperatively acute atrial fibrillation occurred, but could be controlled with digoxin plus sotalol. Digoxin was discontinued after 1 more day, and the patient remained on sotalol $2 \times 40 \mathrm{mg} /$ day. One week later, she had a cardiopulmonary arrest, which was successfully managed by chest compression. The ECG immediately after cardiopulmonary resuscitation showed a bradycardia of $44-52 / \mathrm{min}$, with a QTc of $540 \mathrm{~ms}$. After sotalol was stopped, the heart rate and QTc interval normalized during the following 3 days.

\section{Discussion}

The side-effects of sotalol are related either to its $\beta$ blocking properties or to its propensity to lengthen the QT interval. Adverse reactions due to $\beta$-adrenergic receptor blockade are similar to those associated with conventional $\beta$-adrenergic antagonists. However, aggravation of congestive heart failure by sotalol 


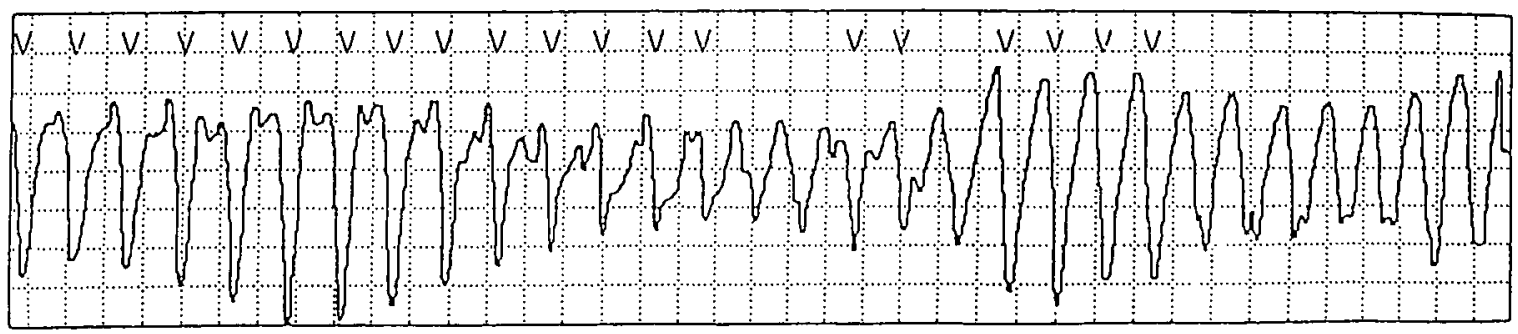

Fig. 1. Electrocardiogram demonstrating torsade de pointes. It is readily apparent that a long pause precedes the sinus beat which triggers a run of polymorphic ventricular tachycardia. The tachycardia is characterized by periodic shifts in the QRS axis and in its amplitude.

is less frequent than might be expected with a $\beta$ adrenergic antagonist.

The most dangerous effect of antiarrhythmic drugs is aggravation of pre-existing arrhythmias or the provocation of new arrhythmias. The drugs that prolong the action potential duration may cause torsade de pointes, a polymorphic ventricular tachycardia associated with an exaggerated prolongation of the QT interval. Many cases of sotalol-induced torsade de pointes have been associated with drug overdose or predisposing factors, such as bradycardia, hypokalaemia, or concomitant use of drugs that prolong repolarization [4-6]. A recent review of data from controlled trials of sotalol in a total of 3257 patients reported proarrhythmic events in $141(4.3 \%)$ patients. Of these, $78(2.4 \%)$ had torsades de pointes, which were more prevalent in patients with congestive heart failure and low ejection fraction. Torsade de pointes were observed early in the course of the treatment and their occurrence was related to. dose [1]. Most studies have reported control of arrhythmia with daily doses between 160 and $640 \mathrm{mg}$ in patients with normal renal function. With doses ranging from 160 to $640 \mathrm{mg} / \mathrm{d}$, the QT interval is prolonged by $40-100 \mathrm{~ms}$. Exaggerated prolongation results in a predisposition to torsade de pointes. Although there are no data from controlled studies, the current recommendation is that sotalol therapy should be discontinued at a maximal QTc interval of $500 \mathrm{~ms}$ [7].

Oral administered sotalol is almost completely absorbed and does not undergo first-pass metabolism in the liver, resulting in a bioavailability of $90-100 \%$; $85-90 \%$ of the drug is excreted unchanged in the urine, the mean elimination half-life being $8 \pm 3 \mathrm{~h}$ in patients with normal renal function. In ESRD the elimination half-life is increased to $41 \mathrm{~h}$, so that a dose reduction to $25 \%$ of the normal dosage is recommended. However, sotalol is effectively removed by haemodialysis (HD), and a supplemental dose of $80 \mathrm{mg}$ is currently recommended after each HD $[7,8]$.

Our three patients were all treated with $80 \mathrm{mg}$ sotalol daily, without drug supplementation after HD, which implies a low dosage of sotalol. Nonetheless cardiopulmonary arrest occurred 1-14 days after the drug had been started. In all three patients, the QTc interval at the time of cardiopulmonary resuscitation was over $480 \mathrm{~ms}$. Although torsade de pointes had been documented in patients 1 and 2 only, the excessive bradycar- dia and prolongation of the QTc interval in patient 3 makes the same diagnosis very probable. All patients had normal electrolytes at the time of cardiopulmonary resuscitation, and none had concomitant therapy with any drug known to prolong cardiac repolarization. Patients 2 and 3, however, had a reduced cardiac ejection fraction.

To our knowledge, this life-threatening complication of sotalol has never been reported before in HD patients. Because of our own experience, we advise extreme caution in the indications and use of sotalol in that special patient population. A daily dose of $40 \mathrm{mg}$ schould not be exceeded in the first days, and daily ECG controls are warranted in the acute titration phase in order to register a possible excessive prolongation of the QTc interval, and stop sotalol medication on time.

\section{References}

1. MacNeil DJ, Davies RO, Deitchman D. Clinical safety profile of sotalol in the treatment of arrhythmias. Am J Cardiol 1993; 72: $44 \mathrm{~A}-50 \mathrm{~A}$

2. Kehoe RF, MacNeil DJ, Zheutlin TA et al. Safety and efficacy of oral sotalol for sustained ventricular tachyarrhythmias refractory to other antiarrythmic agents. $A m J$ Cardiol 1993; 72: $56 \mathrm{~A}-66 \mathrm{~A}$

3. Singh BN, Kehoe R, Woolsley RL, Scheinman M, Quart B. Multicenter trial of sotalol compared with procainamide in the suppression of inducible ventricular tachycardia: a double-blind, randomized parallel evaluation. Sotalol Multicenter Study Group. Am Heart $J$ 1995; 129: 87-97

4. Neuvonen PJ, Elonen E, Vuorenmaa T, Laakso M. Prolonged Q-T interval and severe tachyarrhythmias, common features of sotalol intoxication. Eur J Clin Pharmacol 1981; 20: 85-89

5. McKibbin JK, Pocock WA, Barlow JB, Millar RNS, Obel WP. Sotalol, hypokalaemia, syncope, and torsade de pointes. $\mathrm{Br}$ Heart $J$ 1984; 51: 157-162

6. Krapf $R$, Gertsch $M$. Torsade de pointes induced by sotalol despite therapeutic plasma concentrations. Br Med J 1985; 290; 1784-1785

7. Hohnloser SH, Woosley RL. Drug therapy: Sotalol. $N$ Eng/ J Med 1994; 331: 31-38

8. Bennett WM, Aronoff GR, Golper TA, Morrison G, Brater DC, Singer I. Drug Prescribing in Renal Failure. 3rd edn. ACP, Philadelphia, 1994; 45

Received for publication: 27.11 .95

Accepted in revised form: 7.2 .96

\section{Editor's note}

Please see also the Editorial Comment by Bährle (pp. 944-946 in this issue). 